\title{
Aerodynamic Optimization of the Expansion Section in a Hypersonic Quiet Nozzle Based on Favorable Pressure Effect
}

\author{
Jian Gong, Dapeng Yao*, Xunhua Liu \\ China Academy of Aerospace Aerodynamics, Beijing, 100074, China \\ Email: ${ }^{*}$ ivezaku@gmail.com
}

Received March 2014

\begin{abstract}
Maximum expansion angle is the primary parameter for the design of expansion section of hypersonic quiet nozzle. According to the quantity of maximum expansion angle, expansion section could be classified as fast expansion and slow expansion. In order to diminish the effect of instability of Görtler vortex, gradually, slow expansion was employed for quiet nozzle design. Based on the favorable pressure effect, the maximum expansion angle is optimized in this paper, and a considerable selective session of maximum expansion angle is obtained. The trend that slow expansion is employed instead of fast expansion is explained, and a new method is established for aerodynamic optimization of expansion section contour in a quiet nozzle.
\end{abstract}

\section{Keywords}

Quiet Nozzle, Maximum Expansion Angle, Favorable Pressure Effect

\section{Introduction}

A larger maximum expansion angle was employed in the design of quiet nozzle in the past [1]. In the early 1970 s, a Mach 5 quiet nozzle [2] of which the maximum expansion angle was $22.6^{\circ}$, was preliminarily developed for a leading tunnel at NASA LRC (Langley Research Center). A Mach 3.5 2D quiet nozzle [3] was developed from late 1970s to early 1980s, and its maximum expansion angle of upper surface was $28.75^{\circ}$.

In the early 1990s, a Mach 6 quiet nozzle [4] with a maximum expansion angle of $9.84^{\circ}$, was developed at NASA LRC by employing slow expansion. And in late 1990s, a quiet nozzle with slower expansion was developed at Purdue University [5], and its maximum expansion angle was down to $4^{\circ}$. A Mach 4 quiet nozzle with a maximum expansion angle of $15^{\circ}$ was developed by Zhou Yongwei and Yi Shihe [6] at National University of Defense Technology in 2011. In the course that the fast expansion used in the prior study was gradually replaced by slow expansion in the later term, the knowledge of primary factors for quiet nozzle boundary layer stability was improved [7]. The occurrence of Görtler vortex instability could be delayed by slow expansion [8]. It was noted by Beckwith [9] that the Görtler vortex instability in a contoured nozzle is the main factor of flow insta-

${ }^{*}$ Corresponding author.

How to cite this paper: Gong, J., Yao, D.P. and Liu, X.H. (2014) Aerodynamic Optimization of the Expansion Section in a Hypersonic Quiet Nozzle Based on Favorable Pressure Effect. Journal of Applied Mathematics and Physics, 2, 443-448. 
bility when Mach number is larger than 2.5, and it could be efficiently retarded by slow expansion. It was also suggested that favorable pressure should be considered in the analysis of nozzle boundary layer stability.

In the design of Mach 6 quiet nozzle by Schneider [10], quiet flow condition could be met for both nozzles (one is $240 \mathrm{~mm}$ in diameter of exit and $2.56 \mathrm{~m}$ in length, while the other one is $600 \mathrm{~mm}$ in diameter of exit and $10.36 \mathrm{~m}$ in length) with a maximum expansion angle of $4^{\circ}$, using $\mathrm{e}^{\mathrm{N}}$ transition estimation at a certain unit Reynolds number. However, the result of this semi-theoretic method should be calibrated in wind tunnel tests. Thus, a concept of favorable pressure effect is utilized to study the effect of maximum expansion angle on the pressure gradient on the wall along flow in this paper.

\section{Computation Condition}

To study the effect of maximum expansion angle on the flow field, 7 contours of axial symmetric nozzle with different maximum expansion angles are designed for comparison. According to the improvement of maximum expansion angle, 7 maximum expansion angles between $3^{\circ}$ and $15^{\circ}$ are selected for analysis. All the nozzlesare the same in diameter of exit and Mach number. The main designed configuration parameters of those expansion sections of nozzles are listed in Table 1. It is clear that the length of expansion section increase with maximum expansion angle.

Those 7 contours are also shown in Figure 1. The throat of each nozzle is smoothly connected to the uniformed contraction section. It is clear that the radius of throat is determined with given Mach number and diameter of exit, while the length of expansion section varies with the maximum expansion angle. The points plotted in Figure 2 show the regularity that the length of expansion section decreases with increasing maximum expansion angle.

Figure 2 only describes the relationship between the length of expansion section and maximum expansion angle discretely, and a function in the form of $\operatorname{Ltg} \theta$ could be introduced to demonstrate that in Figure 3. From

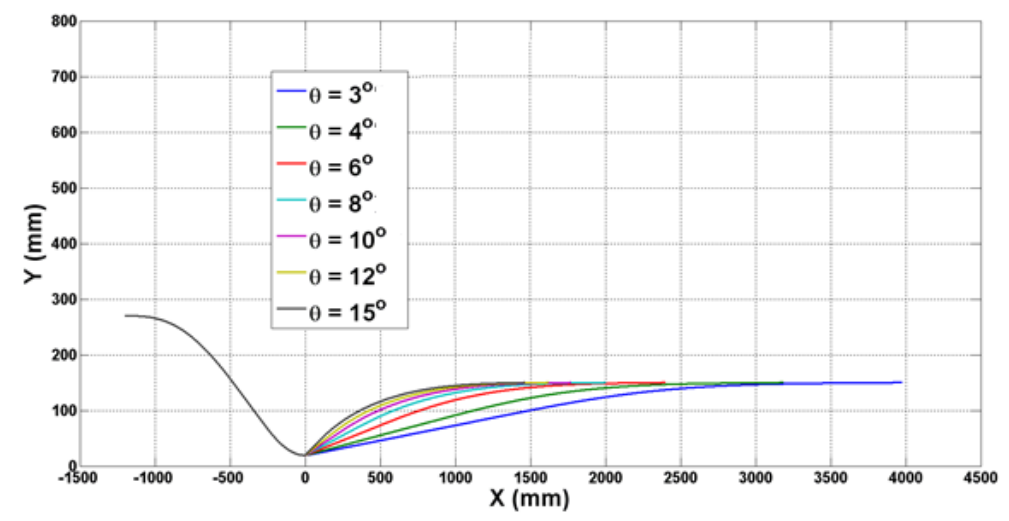

Figure 1. The contours of different axial symmetric nozzle.

Table 1. Configuration parameters of axial symmetric nozzles with the exit Mach number of 6 .

\begin{tabular}{ccccc}
\hline expansion angle & radius of throat & diameter of exit & length of expansion section & length of contraction section \\
\hline 3 & 19.472 & 300 & 3976.844 & 1200 \\
4 & 19.472 & 300 & 3186.006 & 1200 \\
6 & 19.472 & 300 & 2400.502 & 1200 \\
8 & 19.472 & 300 & 2007.019 & 1200 \\
10 & 19.472 & 300 & 1771.169 & 1200 \\
12 & 19.472 & 300 & 1621.662 & 1200 \\
15 & 19.472 & 300 & 1464.190 & 1200 \\
\hline
\end{tabular}




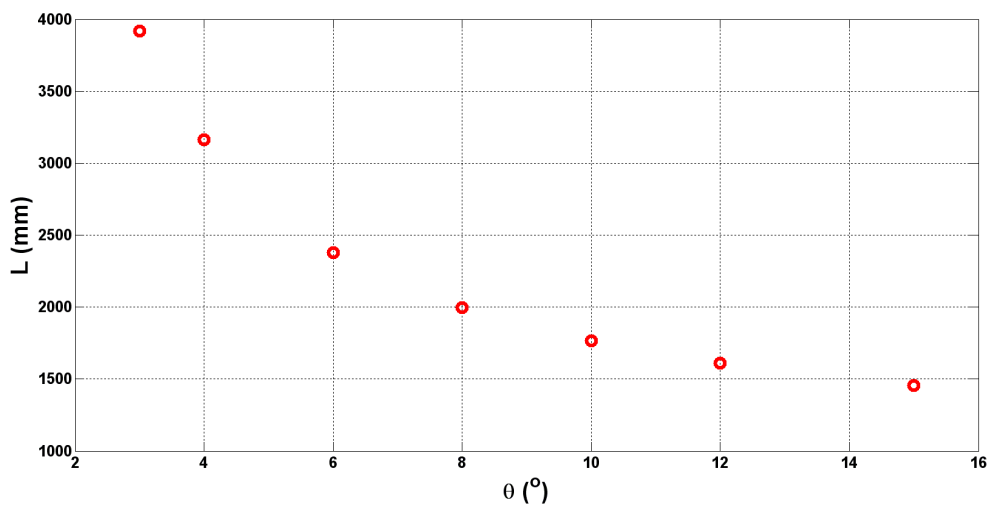

Figure 2. The relationship between the length of expansion section and maximum expansion angle.

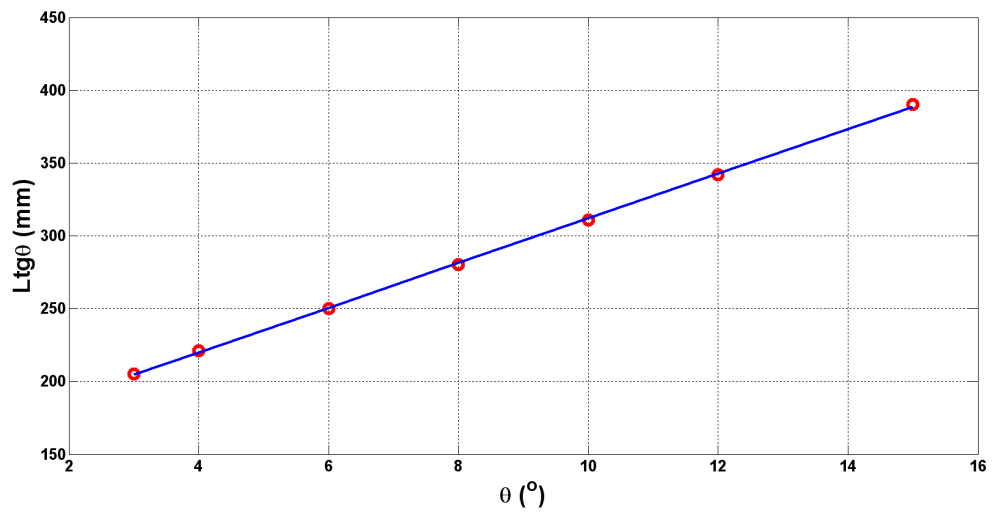

Figure 3. $\operatorname{Ltg} \theta$ varies as a function of maximum expansion angle.

the fitted line, it is noted that $\operatorname{Ltg} \theta$ is linear to the maximum expansion angle $\theta$. The function can be described as:

$$
\operatorname{Ltg} \theta=k \theta+b
$$

where, $k$ and $b$ are coefficients of the equation.

Substituting the data in Table 1 into the equation above and using least square fit, the coefficients are determined:

$$
\operatorname{Ltg} \theta=15.326 \theta+158.734
$$

The relationship between the length of expansion section and maximum expansion angle is defined by this equation. Thus, one of those two terms can be estimated using this equation in nozzle design given that the quantity of the other is determined. Additionally, a too long nozzle will lead two negative results: the increased difficulty in machining the inner surface and the easier transition in the boundary layer of nozzle wall cause by T-S instability. Thus, the value of maximum expansion angle should not be selected too small.

\section{Flow Field Computation}

Flow fields near the throats of nozzles with different maximum expansion angle are shown in Figure 4. It is noted that the change of pressure gradient becomes faster in the downstream flow field when maximum expansion angle increases, given the same contraction section. Although all the nozzles finally provide flow of good uniformity, it is critical to keep pressure gradient changing slowly for quiet nozzle.

The relationship between the distance that a point with a certain Mach number longitudinally departs from throat and maximum expansion angle is shown in Figure 5. The longitudinal gradient of Mach number increases with maximum expansion angle. 


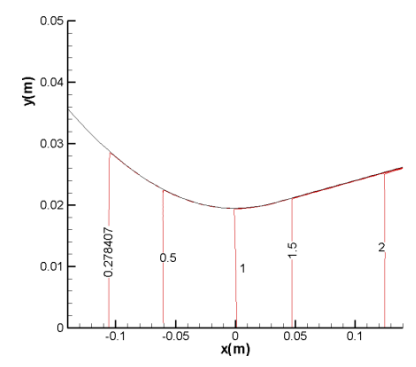

(a)

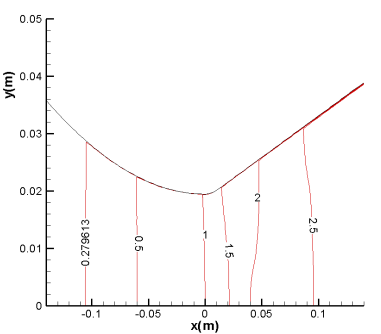

(d)

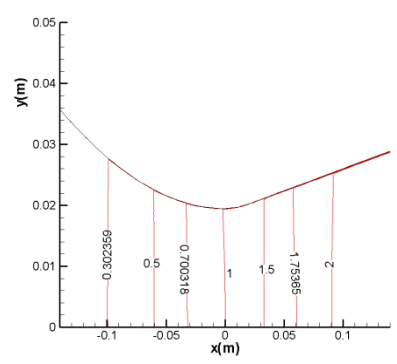

(b)

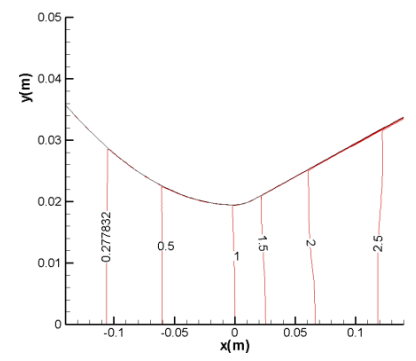

(c)

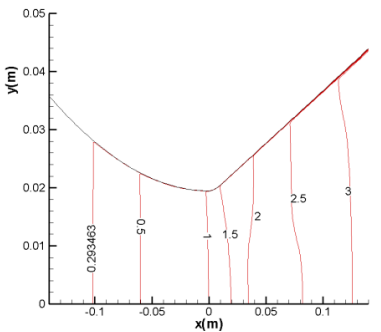

(e)

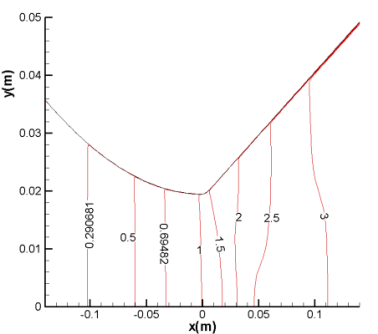

(f)

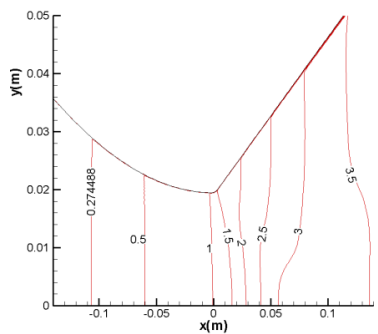

(g)

Figure 4. Mach number contours around the throat for various maximum expansion angle. (a) $\theta=3^{\circ}$; (b) $\theta=4^{\circ}$; (c) $\theta=6^{\circ}$; (d) $\theta=8^{\circ}$; (e) $\theta=10^{\circ}$; (f) $\theta=12^{\circ}$; (g) $\theta=15^{\circ}$.

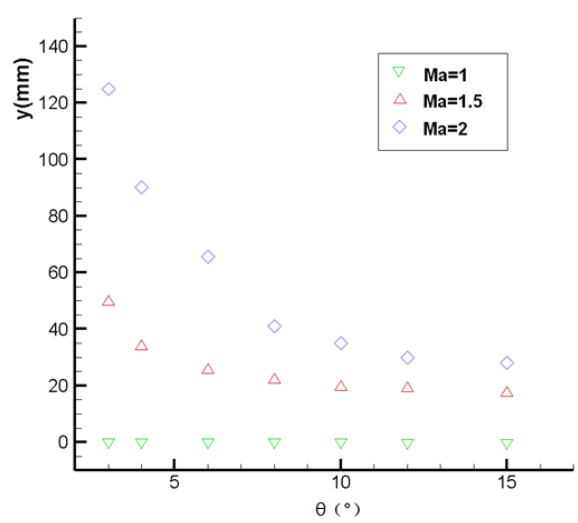

Figure 5. The relationship between the distance that a point with a certain Mach number longitudinally departs from throat and maximum expansion angle.

The distributions of Mach number at nozzle exit in the condition of different maximum expansion angles are shown in Figure 6. The flow field at exit for each maximum expansion angle is of good uniformity. The distribution of downstream pressure on the inner wall of each nozzle is shown in Figure 7. Generally, the pressure on the wall continues to decrease, demonstrating that flow field near the wall in the nozzle is in a status of favorable pressure. The decreasing increscent of pressure on throat wall increases with maximum expansion angle. For the design of nozzle contour, this favorable pressure indicates the increase of initial force in the flow, which would diminish the strength and propagation of disturbance, and eventually help prevent the transition of boundary layer.

Based on the results given by Figure 7, the distributions of downstream pressure gradients on the walls in the whole range of contoured nozzles are shown in Figure 8. It is noted that the distributional differences are mainly in the area near the throat. Thus, the distributions of pressure gradients on the walls near the throat are demonstrated in Figure 9.

Figure 8 shows that downstream pressure gradients are all minus, further indicating the fact that pressures on the walls continue to decrease. Also, peak values of downstream pressure gradients on the walls, which mean most significant changes in pressure, are reached near the throats. 


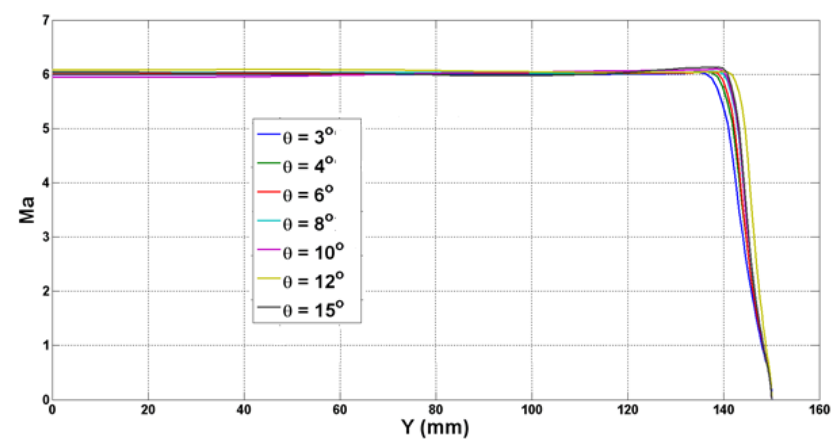

Figure 6. The distributions of Mach number at nozzle exit for various maximum expansion angle.

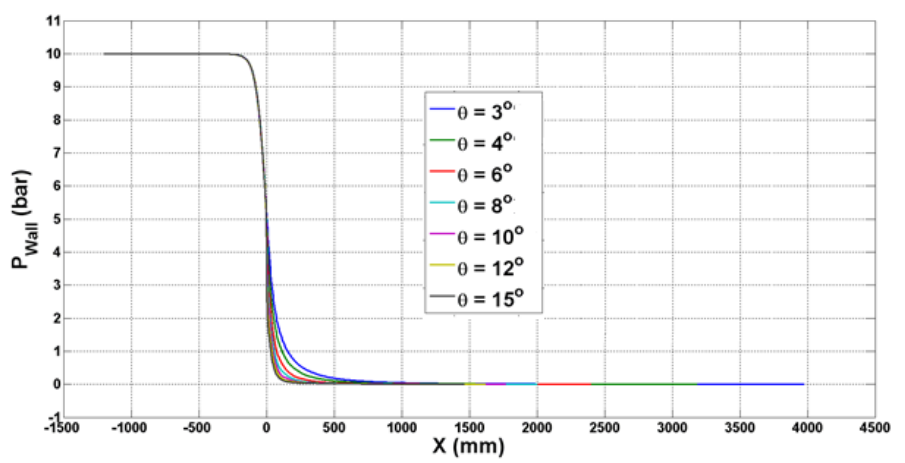

Figure 7. The distributions of static pressure on the nozzle wall.

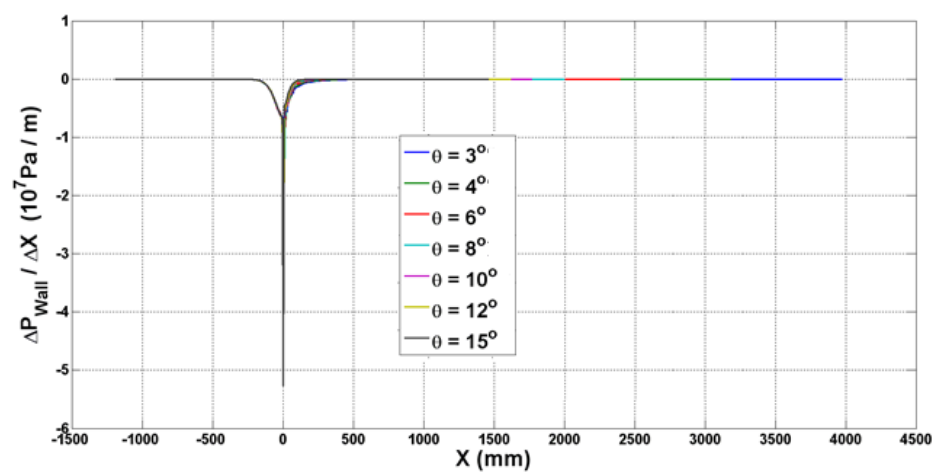

Figure 8. The streamwise distribution of static pressure on the nozzle wall.

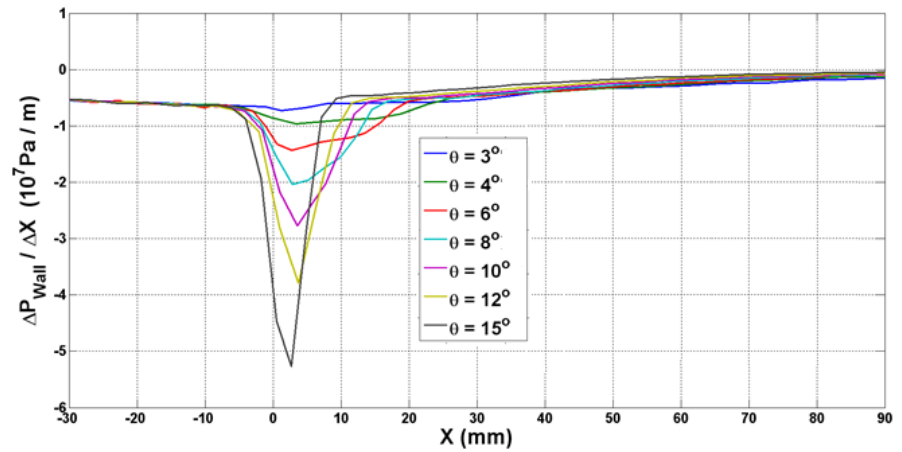

Figure 9. The distribution of pressure gradient on the nozzle wall for various maximum expansion angle. 
As shown in Figure 9, in the range $3-5 \mathrm{~mm}$ downstream from throat, peak values of downstream pressure gradients are reached for all the nozzles with different maximum expansion angles. The absolute value of peak pressure gradient decreases with maximum expansion angles, indicating that the change of wall pressure becomes mitigated. Because it is convex wall for nozzle flow near the throat, the low favorable pressure drives flow moving downstream steady rather than rapid expansion which would lead to oscillation in flow and nozzle wall.

\section{Conclusion}

It can be concluded that a lower maximum expansion angle is required for quiet nozzle. However, a too low value is also unfeasible because it may lead to extremely long nozzle consisting of more sections. Specifically, if the maximum expansion angle is set as $3^{\circ}$, a $4 \mathrm{~m}$ long expansion section which must be divided into more than 10 sections would be required (the $4^{\circ}$ quiet nozzle at Purdue University is $2.56 \mathrm{~m}$ long and consists of 8 sections). Difficulties would be encountered in machining to meet the requirement of low roughness and smooth joint. in another words, maximum expansion angle should be neither too large nor too small, and a optimized value is to be determined. According to the discussion in the previous part, a optimized range of maximum expansion angle is $4^{\circ}-6^{\circ}$. The fact that the maximum expansion angle of the Mach 6 quiet nozzle (the longest nozzle all over the world) designed by Schneider is $4^{\circ}$, agrees with our analysis.

\section{References}

[1] Beckwith, I.E. (1975) Development of a High Reynolds Number Quiet Tunnel for Transition Research. AIAA Journal, 13, 300-306. http://dx.doi.org/10.2514/3.49695

[2] Anders, J.B., Stainback, P.C. and Beckwith, I.E. (1978) A New Technique for Reducing Test Section Noise in Supersonic Wind Tunnels. AIAA Paper: 1978-0817.

[3] Beckwith, I.E. and Moore, W.O. (1982) Mean Flow and Noise Measurements in a Mach 3.5 Pilot Quiet Tunnel. AIAA Paper: 1982-0569.

[4] Chen, F.J., Wilkinson, S.P. and Beckwith, I.E. (1991) Görtler Instability and Hypersonic Quiet Nozzle Design. AIAA Paper: 1991-1648.

[5] Schneider, S.P. (1998) Design and Fabrication of a 9.5-inch Mach-6 Quiet-Flow Ludwieg Tube. AIAA Paper: 1998-2511.

[6] Zhou, Y.W., Chang, X.Y., Yi, S.H. and Zhang, Y. (2002) The Aerodynamic Design of Supersonic Quiet Wind Tunnel. Experiments and Measurements in Fluid Mechanics, 16, 61-66.

[7] Schneider, S.P. (2008) Development of Hypersonic Quiet Tunnels. Journal of Spacecraft and Rockets, 45, 641-664. http://dx.doi.org/10.2514/1.34489

[8] Zhou, Y.W. and Yi, S.H. (2010) Summarization of the Characteristics and Development of Hypersonic Quiet Wind Tunnel. Journal of Experimental Mechanics, 25, 167-172.

[9] Beckwith, I.E. and Holley, B.B. (1981) Görtler Vortices and Transition in Wall Boundary Layers of Two Mach-5 Nozzles. NASA-TP-1869.

[10] Schneider, S.P. (1998) Design of a Mach-6 Quiet-Flow Wind-Tunnel Nozzle Using the eN Method for Transition Estimation. AIAA Paper: 1998-0547. 